\title{
MMW-HOTLINE
}

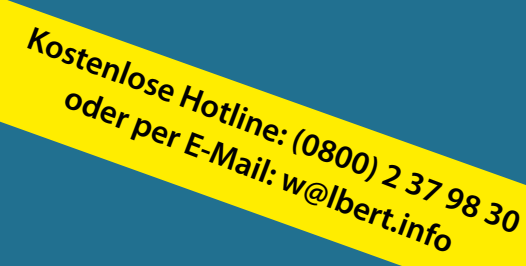

Leser der MMW können sich mit allen Fragen zur Abrechnung und Praxisführung an Helmut Walbert, Facharzt für Allgemeinmedizin, Würzburg, wenden. Sie erreichen ihn jeden Donnerstag von 13 bis $15 \mathrm{Uhr}$ unter der kostenlosen Rufnummer (0800) 2379830 oder per E-Mail: w@lbert.info.

\section{Gilt da jetzt die Vertreter- oder die Versichertenpauschale?}

Dr.med. J. S., Allgemeinärztin in Z.:

Ein Patient, der bisher bei einem anderen Allgemeinarzt in Behandlung war, wechselt zu uns. Rechnen wir für das laufende Quartal die Vertreter- oder die Versichertenpauschale $a b$ ?

Antwort: Die Versichertenpauschalen 03120 bis 03122 bei Überweisung oder einer Behandlung im Vertretungsfall kommen genau nur in diesen Fällen zum Tragen! Haben Sie keine offizielle, der KV gemeldete Vertretung oder kommt ein Patient einfach so - wie oben angefragt - in die Praxis und möchte behandelt werden, fällt die normale Versichertenpauschale nach GOP 03110 bis 03112 an. Dies ist vor allem betriebswirtschaftlich von Bedeutung, da die normale Versichertenpauschale deutlich höher - fast doppelt so hoch - vergütet wird. Bis zum Jahresanfang war die anfallende Kassengebühr eine gewisse Bremse. Eine mehrfache Inanspruchnahme von Ärzten der gleichen Fachgruppe durch denselben Patienten wird in Zukunft aber wieder häufiger der Fall sein.

\section{Wie wird das privat abgerechnet?}

Dr.med. K.-H.S, Allgemeinarzt, München: Bei Privatpatienten mache ich neben einem Check-up häufig auch ein Hautkrebsscreening (HKS). Wie kann ich diese Leistungskombination abrechnen?

Antwort: Im Prinzip sollte man diese Leistungskombination aus abrechnungstechnischen Gründen vermeiden, weil die augenblickliche GOÄ keine leistungsgerechte Abrechnung des Hautkrebsscreenings (HKS) neben einem Check-up erlaubt. Daher ja wohl auch die Frage.

Ein HKS als alleinige Leistung zu einem Konsultationszeitpunkt wird nach GOP 1 „Beratung bis 10 Minuten", GOP 7, „Untersuchung des Hautorgans" und - falls vorhanden "Untersuchung mit dem Auflichtmikroskop", GOP 750 abgerechnet, also insgesamt 380
Punkte, $€ 48,26$. Da die "Beratung" umfassend über Vorsorge, Sonnenschutz, Eigenbeobachtung usw. erfolgen sollte, reicht die Beratungszeit „bis zehn Minuten“ normalerweise nicht aus. Es käme also die GOP 3 zum Ansatz. Allerdings ist dann die Abrechnung der GOP 750 ausgeschlossen. GOP 3 käme also nur für die Praxen in Frage, die die GOP 750 nicht erbringen. Demzufolge muss für eine leistungsgerechte Abrechnung die GOP 1 auf 3,5-fach, $€ 16,32$, gesteigert werden mit der Begründung: „Erheblicher Zeitaufwand bei notwendiger Beratung über zehn Minuten". Da neben dem Check-up, GOP 29 die GOP 3 und 7 ausgeschlossen sind, sollte man organisatorisch die Untersuchungen trennen, um ein leistungsgerechtes Honorar erarbeiten zu können.
Versicherungsanfrage

\section{Euro sind ein Witz}

Dr.med. U. T., Allgemeinmedizin, KVB: Muss ich bei Versicherungsanfragen (als Bericht nach GOP 75) eine Vergütung von $€ 20$ akzeptieren? Das ist meines Erachtens keinesfalls kostendeckend.

Antwort: Nein! In der Regel werden Sie zu Stellungnahmen aufgefordert wie: Ist der Patient heute gesund? Sind bleibende Folgen zu erwarten? Diese Frage erfüllen den Tatbestand der GOP 80 "Schriftliche gutachtliche Äußerung". Diese GOP ist mit $€ 40,22,2,3$-fach bewertet. Hinzu kommt bei GOP 80 die GOP 95 Schreibgebühr je DIN A4-Seite sowie die GOP 96, Schreibgebühr je Kopie $€ 0,17$, auch für Kopien, die für die eigenen Unterlagen gebraucht werden! Vorprogrammierter Ärger mit der Versicherung kann weitestgehend vermieden werden, indem am besten taggleich mit der Anforderung Ihrerseits eine Kostenübernahmeerklärung per Fax herausgeht. Sie versichern nach Eingang der Kostenübernahme, innerhalb von 48 Stunden die Anfrage zu beantworten. Dies bewirkt bei vielen Versicherern - die gewohnt sind, oft wochenlang zu warten und mehrmals anzumahnen - Wunder! Gleichzeitig teilen Sie mit, dass Sie nach Ablauf von zehn Tagen die Anfrage als gegenstandslos "schreddern“. Bleiben Sie kostenbewusst! Leider ist bei vielen Kolleginnen und Kollegen auf Grund der miesen Vergütung in der GKV die persönliche Wertschätzung der eigenen Leistung verloren gegangen. Als Unternehmer Arzt sollten wir wenigstens $€ 250$ brutto pro Stunde erarbeiten! 